\title{
We Are Not the Same Either Playing: A Proposal for Adaptive Gamification
}

\author{
Inmaculada RODRÍGUEZ ${ }^{\mathrm{a}}$, Anna PUIG ${ }^{\mathrm{a}}$ and Alex RODRÍGUEZ ${ }^{\mathrm{a}} 1$ \\ ${ }^{a}$ Department de Matemàtiques i Informàtica, IMUB and UBICS Research Institutes, \\ Universitat de Barcelona, Spain
}

\begin{abstract}
Gamification consists in applying game mechanics in non-game contexts aiming at motivating and shaping behaviours. This paper proposes an adaptive approach for gamification, which takes as initial information players profiles - gathered from Hexad player type questionnaire - and considers also how these profiles change over time based on users interactions. Then, we provide the users with a personalised experience through the use of game elements that correspond to their dynamic playing profile. We present a preliminary evaluation of the approach by means of a simulator that yields promising results when comparing it with baseline configurations, i.e randomized and fixed player profile.
\end{abstract}

Keywords. adaptive gamification, dynamic player type

\section{Introduction}

Gamification applies elements of game playing to non-game contexts to pursue an objective while increasing user engagement and motivation. This objective varies widely depending on the context. For example, a gamified fair [21] was designed to provide visitants with the best experience visiting the fair. Gamification in MOOCs (Massive Open Online Course) aims to foster students' engagement and therefore increase the completion rate of courses [9]. In the bussiness context, the gamification of e-marketing processes aims to increase the site visits and sales [24].

Whatever the goal, gamification comes into the picture to encourage users behaviours through gameful experiences. Nevertheless, gamification designs usually take the one-fits-all approach, which may fail as result of considering that all users have the same profile. The opposite approach is adaptive gamification which takes into account userss diversity, i.e. the users are driven by different motivations.

Adaptive gamification found its basis on player types classifications. Nevertheless, whenever gamification has been approached from the adaptive perspective, it has been done gathering information about the user profile (i.e player types) fixed at the beginning of the process [16]. To do so, users answer a player type questionnaire, then the gamified system proposes the users game elements (e.g. badges, challenges) tailored to their pro-

\footnotetext{
${ }^{1}$ Research supported by Accio COMRDI18-1-0010, MISMIS-Language (PGC2018-096212-B-C33) and CI-SUSTAIN (PID2019-104156GB-I00).
} 
files. However, these approaches have some limitations. This initial player profile either may have been provided by the user inaccurately or can slightly change/evolve along the time [5]. Additionally, adaptive gamified systems usually take the most predominant player type to show the user game elements related to this predominant player type, with the drawback of ignoring other player types that also may characterise the user, so that it should be considered several player types, not only the predominant one [13].

In this paper, we propose an adaptive approach for gamification to find the most suitable game elements for the player type of the user. Our approach uses initial players profiles, gathered from Hexad questionnaire [12], and further information about users interactions while using the system. Specifically, we consider user's interactions with game elements and user's opinions about those elements. Gamification goal is fostering users' engagement and so motivate the completion of online activities such as learning activities in a course or employees progress report in a company. Last but not least is to comment about the evaluation of the system. Although evaluations of the effects of gamification are commonly carried out with users, this paper presents a previous analysis of the proposed strategy using bots. The bots simulate different types of users, not so much to evaluate the effects of gamification (i.e. completion rate), but to validate the convergence and validity of our method.

\section{Previous work}

Recently, techniques user-centered have been proposed to correlate the game elements to different user profiles [12][14]. Some of them focused on specific user characteristics, such as motivation [4], personality treats [7] [8], learning styles [3], player types [16], or type of interaction with different activities [15]. Others combined different characteristics, such as [6] that took into account Learning Styles and Player Typesto end up determining the types of educational activities and game elements to include in a learning pathway. In contrast, [18] suggested using context, interactions, gender and player type to decide, through rules, the next game element to display. Other works focused on emotions to predict the individual's performance on gamified tasks, information that could potentially be used to adapt the game features [17].

Specifically, in current adaptive gamification approaches, the most commonly used taxonomies of player types have been Bartle's [2], BrainHex [20] and Hexad [1]. These taxonomies allow to identify player types easily from questionnaires and also allow to establish the correspondence between these Player Types and the Game Elements [14]. Questionnaires allow characterising the Player Type of a user with a set of ratings. They determine the player type prior to the experience. For example, the HEXAD model distinguishes between 6 player's types (Achiever, Player, Philanthropist, Disruptor, Socializer and Free Spirit), then as result of the questionnaire the user could obtain the ratings: Achiever 25\%, Player 18\%, Philanthropist 21\%, Disruptor 8\%, Socializer $10 \%$ and Free Spirit 5\%. The decision of the final Player Type and thus, the most appropriate game element, usually relies on the predominant rating [16] or some combination of them [19]. In this work, we rely on the HEXAD player model and use the questionnaire proposed and validated by [23]. However, it should be noted that in all the studies analysed, the No-Player type of player is not considered, a fact that, in some cases, the use of gamification is more detrimental than beneficial [11]. Thus, we also add the No-Player player type to the HEXAD taxonomy, and also we consider for each user the assessment of all 
their ratings included in her Player Type.

Certainly, as supported by different studies [22], the interpretation of the results of these questionnaires may not be very reliable. Even if the questionnaire is valid, the answers may be somewhat random or the results at the beginning may not persistent during the experience depending on the moment or the mood the user. In fact, in addition to questionnaires, some proposals in the literature also gathered user feedback of learning activities [15], or scores on different game elements [10] during the experience. Thus, inspired by these works, we enrich the user model obtained from the initial questionnaire by means of user interactions and opinions during the course of the activity. Therefore, we will base the adaptation of the game mechanics to the "real" and "dynamic" user profile using two types of interactions: on the one hand, the interactions with the game elements and, on the other hand, the opinions that the user can make at certain moments about those elements. Thus, using both types of interactions, we will refine the players types during the experience, and, consequently, the game elements to be activated.

Regarding adaptive strategies proposed in the literature, most of them are more concerned with personalisation of learning rather than gamification per se [12]. In the following we refer to those that concretely focused on adapting gamification. [16] proposed a matrix factorisation model similar to those used in recommender systems. They used two matrices: one defining the player types of all users and the other representing how the game elements match the player types. They combined these two matrices to obtain game elements' scores for each user, and then they selected the element with the highest score. On the other hand, [6] defined an off-line Q-Learning algorithm to generate an adaptive learning path of the user. They defined S-Table and Q-Table that represented the correspondent state at each taken action, and the Q-values of each action in each state, respectively. Both tables were the same for all the profiles. Nevertheless, R-Table was specific for each Learning-Player profile. All of them consider adapting game elements to the initial player's profile, keeping the player' type static throughout the experience.

To the best of our knowledge our approach is the first that takes a dynamic picture of the user experience. Our adaptive algorithm is based on a matrix factorisation model similar to [16] that allows the recalculation of the player type, i.e. of all player ratings, during the experience in order to adapt the game element to the user model at any given moment. In this initial study, adaptation is based on activating one of the most appropriate game element at any time depending on the recalculated Player Type.

\section{Runtime method for adaptive gamification}

In this section we present our proposal for adaptive gamification. First, we introduce the main definitions our method is based on: (i) the Hexad player type model, including the non-player type, (ii) the selected game elements as a subset of those proposed by Tondello [23], and (iii) we define the matrices and vectors of ratings and interactions implied in our algorithm. Second, we describe how we match these player types with their corresponding game elements using an extended matrix factorisation method [16].

\subsection{Previous definitions}

\section{Player type}

An essential concept for adaptive gamification is the player type model, which classifies what kind of game elements maximise user motivation. As we mentioned above, we base 
on the Hexad player typology, adding the non-player type [1]. Player types are defined as:

$$
P T=\left\{p t_{1}, p t_{2}, \ldots, p t_{7}\right\}
$$

The player types, $p t_{i}$ are explained below:

1. Disruptor: Motivated by the ability to modify the system.

2. Free Spirit: Motivated by the ability to freely explore the system.

3. Achiever: Motivated by the ability to win challenges and unlock hidden content.

4. Player: Motivated by the game itself.

5. Philanthropist: Motivated by the ability to share goods and help other users.

6. Socializer: Motivated by social connections.

7. Non-Player: Users who don't like to play.

The player type of a particular user is represented as a vector of length $7, P R$, where each component $r_{i}$ represents its ratings for each player type, so the values vary between 0 and $1, P R=\left(r_{1}, r_{2}, \cdots, r_{7}\right)$. For example, a user can be $20 \%$ Disruptor, $10 \%$ Free Spirit, 30\% Achiever, 40\% Player, 40\% Socializer, 10\% Philanthropist and $0 \%$ No Player, which is encoded with the vector $(0.2,0.1,0.3,0.4,0.4,0.1,0)$. Since the user's player type changes along the time, we define $P R^{(t)}$ as the Player Ratings at the time $t$ such as:

$$
P R^{(t)}=\left(r_{1}^{(t)}, r_{2}{ }^{(t)}, \cdots, r_{7}{ }^{(t)}\right)
$$

\section{Game element}

Based on the correlation analysis of the Hexad player types with 52 game design elements done by [23], we select a subset of 14 game elements covering the whole spectrum of player types (see Figure 1). There are no game element associated to non-player type. Game elements are defined as:

$$
G E=\left(g e_{1}, g e_{2}, \ldots, g e_{14}\right)
$$

The game elements, $g e_{i}$, are briefly explained below:

1. Development Tool: Allows the player user to create some gamification mechanics such as badges, challenges and unlockables.

2. Challenge: The player must overcome a challenge, such as reaching a certain level, solving a problem in a certain time, etc.

3. Easter Egg: The mechanic consists of an image that, when pressed 5 consecutive times, allows access to a mini-game.

4. Unlockable: When a player overcomes a certain challenge, a hidden content is unlocked, which can be a message, a mini-game, etc.

5. Badge: Awarded to the player when she manages to complete a difficult task.

6. Level: Shows the user's progress in completing a task, subdivided into levels.

7. Point: The player gains score, experience, virtual money, etc.

8. Leaderboard: Displays a ranking of scores. 
9. Gift Opener: The player opens gifts she has received.

10. Lottery: Game of chance (roulette) that allows to increase scores of a player.

11. Social Network: Small social network that allows players to create a profile, add friends and view their profile.

12. Social Status: Collection of rankings of players based on their scores, especially those related to social interaction, such as the number of followers, visitors, etc...

13. Share Knowledge: The player sends help messages to everyone in a group.

14. Gift: The player sends gifts to other users.

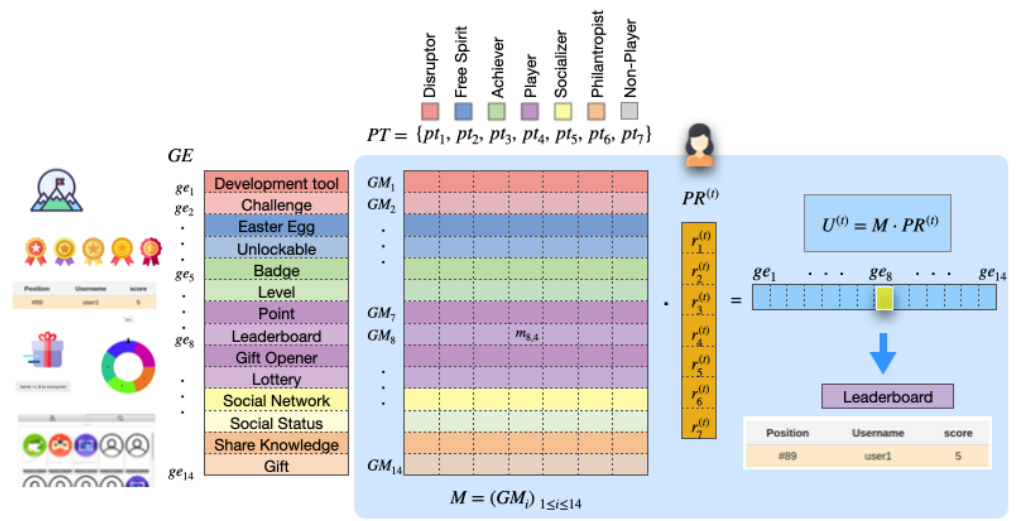

Figure 1. Selected 14 game elements, $g e_{i}$, enumerated by $i=1 . .14$. Each colour represents one Player Type, $p t_{j},(j=1 . .7)$. The vector $P R^{(t)}$ defines the Player Ratings of a user, and the matrix $M$ stores all the motivation values that the $i$-th game element produces to the $j$-th Player Type. The game element having the highest utility is the item that will be presented to the user. As an example, game element 8-th, the leaderboard, is highlighted to represent the next game element to be shown to the user.

It is worth mentioning that each game element does not target only one type of player, but can motivate different types of players. Thus, the $i$-th game element, $g e_{i}$, has associated a vector of motivation indexes $G M_{i}$, where each component, $m_{j}$, is the percentage of motivation it can cause in one of the 7 types of players, $p_{j}$. For example, the fifth game element "Badge" (see Figure 1) can motivate users with both Achiever $\left(p t_{3}\right)$ and Player $\left(p t_{4}\right)$ player types, then the $G M_{5}=(0,0,0.5,0.5,0,0,0,0)$.

$$
G M_{i}=\left(m_{1}, m_{2}, \ldots, m_{7}\right) \forall i=1 \ldots 14
$$

Therefore, we define the matrix, $M$ as:

$$
M=\left(G M_{i}\right)_{1 \leq i \leq 14}=\left(m_{i j}\right)_{1 \leq i \leq 14,1 \leq j \leq 7}
$$

where the rows of this matrix are indexed by the game elements, and the columns by the player types. Thus, $m_{i, j}$ represents the percentage of motivation (motivation index) that the $i$-th game element produces to the $j$-th type of player.

Finally, as can be appreciated on the right side of Figure 1, the utility of using a game element, $U^{(t)}$, can be computed from the matrix $M$ and the player type ratings, $P R^{(t)}$. 


\section{Interaction index}

It is defined as the percentage of user's interaction with each game element at time $t$. This is represented by a vector of length 14 (the number of game elements), $S^{(t)}=$ $\left(s_{1}^{(t)}, s_{2}^{(t)}, \cdots, s_{14}^{(t)}\right)$. The interaction index of the $i$-th game element, $s_{i}^{(t)}$, is defined by

$$
s_{i}^{(t)}=1-e^{-\left(o_{i}^{(t)} \frac{n_{i}^{(t)}-n_{i}^{(t-1)}}{\tau_{i}^{(t)}-\tau_{i}^{(t-1)}}\right)}
$$

where,

$\tau_{i}^{(t)}:$ the Display time i.e. the time interval for which the game element has been displayed until time $t$,

$n_{i}^{(t)}:$ the number of interactions at time $t$,

$o_{i}^{(t)}$ : the Opinion, i.e. user assessment about game element, it's a value between 0 and 1. Opinions from 1 to 5 stars correspond to $0.2,0.4,0.6,0.8$ and 1 respectively.

Note that Equation 6 encodes the interaction index as a number between 0 and 1 . If there are no interactions between time $t$ and $t+1$, the interaction index is 0 (since $\left(n_{i}^{(t)}-n_{i}^{(t-1)}\right)$ is 0$)$. The interaction speed is $\left(\frac{n_{i}^{(t)}-n_{i}^{(t-1)}}{\tau_{i}^{(t)}-\tau_{i}^{(t-1)}}\right)$. Then, the interaction index tends to 1 as the interaction speed increases.

The Opinion $o_{i}^{(t)}$ modulates the interaction speed: $s_{i}^{(t)}$ tends faster to 1 when $o_{i}^{(t)}$ is near 1 than when $o_{i}^{(t)}$ is near 0 .

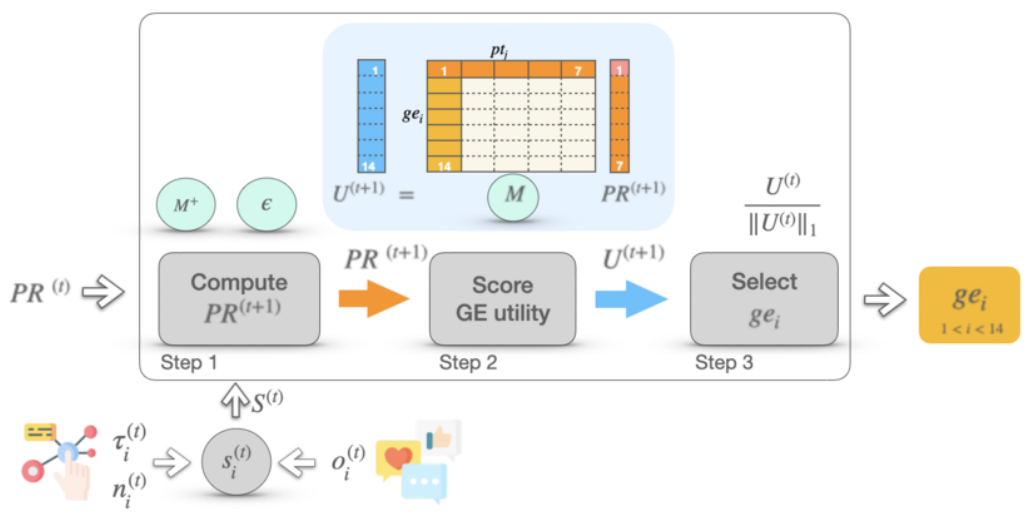

Figure 2. Steps to compute the utility of showing a $g e_{i}$ associated to a user at time $t+1$. Blue circles indicate the constant data of our method. All other elements define dynamic values that change over time.

\subsection{Adaptive method}

We propose an iterative method that calculates the utility of showing a game element to a given user at time $t$. In each iteration, it performs three steps depicted in Figure 2 (considering definitions introduced in section 3.1). The steps are: (1) Obtain $P R^{(t+1)},(2)$ Score the utility of showing a game element to a user at a specific time $t+1$ (denoted by $U^{(t+1)}$ ), and (3) Select which game element to activate based on the assigned scores. 
In the first step, we compute the new player type ratings, $P R^{(t+1)}$ :

$$
P R^{(t+1)}=(1-\varepsilon) P R^{(t)}+\varepsilon\left(M^{+} \cdot S^{(t)}\right)
$$

where,

$P R^{(t)}$ : the player type of the user at time $t$,

$S^{(t)}:$ the interaction indexes,

$\varepsilon$ : to avoid extreme fluctuations between $P R^{(t)}$ and $P R^{(t+1)}$, where $0<\varepsilon<1$. The value of this parameter should be tuned experimentally.

$M^{+}$: the Moore-Penrose pseudoinverse matrix of $M$, needed in order to interpret $S^{(t)}$ and $P R^{(t)}$ in the same space.

In the second, once we have calculated the new player profile, we compute the utilities as indicated in the top right side of Figure 1, using the matrix $M$ defined in Equation 5:

$$
U^{(t+1)}=M \cdot P R^{(t+1)}
$$

Finally, in the third step, we select the next game element to display considering the $i^{t h}$ component of $\frac{U^{(t)}}{\left\|U^{(t)}\right\|_{1}}$ as the probability of choosing the $i^{t h}$ game element using a weighted random choice ${ }^{2}$.

\section{Simulations}

An adaptive gamification strategy works if the game element proposed to the user fits its "real" player profile at any time $t$. Considering that we simulate the player using a bot, in the following, we note the "real" profile of the bot as $R P T_{0}$, and its player type rating at time $t$ as $P R^{(t)}$.

The values of $R P T_{0}$ come from data gathered from user types Hexad test results ${ }^{3}$, where 42782 tests were carried out, obtaining average type scores for all the modalities of players. We selected the eleven most representative modalities (see Table Summary ${ }^{3}$ )) and their corresponding average type scores (see Table Average Type Scores ${ }^{3}$ ). For instance, the Achiever modality appears in the $12 \%$ of the tests and its average scores are $R P T_{0}=(0.12,0.18,0.20,0.16,0.16,0.17,0.0)$ meaning $12 \%$ Disruptor, $18 \%$ Free Spirit, $20 \%$ Achiever, $16 \%$ Player, $16 \%$ Socialiser, $17 \%$ Philanthropist, and $0 \%$ Non-Player.

Keeping in mind that a real user would answer the Hexad questionnaire reliably (accurately) or unreliably (inaccurately) at the beginning of the gamified experience, our bot simulates users' responses accurately or somewhat randomly. Therefore, we define $P R^{(0)}$ being close to $R P T_{0}\left(R P T_{0} \simeq P R^{(0)}\right)$ or far away from $\left(R P T_{0} \nsucceq P R^{(0)}\right)$. To do so, if the reliability is low, the bot rating $P R^{(0)}$ is the furthest non-null scores from $R P T_{0}$ in Table Average Type Scores. Otherwise, if the reliability is high, the bot takes its $P R^{(0)}$ as $R P T_{0}$. Note that when the bot simulates accurate responses to the questionnaire it is desirable that the value of $P R^{(t)}$ remains close to $R P T_{0}$, while when it simulates inaccurate answers it is convenient that $P R^{(t)}$ converges to $R P T_{0}$ when $t \rightarrow \infty$.

\footnotetext{
${ }^{2}\|\cdot\|_{1}$ is the $\ell_{1}$-norm.

${ }^{3}$ https://gamified.uk/UserTypeTest2016/user-type-test-results.php
} 
Besides, the bot interacts with the game elements obtaining interaction indexes, $S^{(t)}$, from $\tau_{i}^{(t)}, n_{i}^{(t)}, o_{i}^{(t)}$ (see input data of $s_{i}^{(t)}$ in the bottom left of Figure 2, and Equation 6). To do so, we use two variables: the time between two consecutive interactions, $\top_{i}^{(t)}$, and the opinion, $\Theta_{i}^{(t)}$, defined as:

$$
\begin{aligned}
\top_{i}^{(t)} & =-9.5\left(G M_{i} \cdot R P T_{0}\right)+10 \\
\Theta_{i}^{(t)} & =\frac{1}{5} \operatorname{round}\left(4\left(G M_{i} \cdot R P T_{0}\right)+1\right)
\end{aligned}
$$

where $i$ corresponds to the game element $\left(g e_{i}\right)$ selected by the method. Thus,

- If the game element $\left(g e_{i}\right)$ fits the real bots profile $\left(R P T_{0}\right)$, the bot interacts more frequently than otherwise. Therefore, $T_{i}^{(t)}$ reflects this behaviour taking values from 0.5 (frequent interactions) to 10 (longer time between interactions).

- Regarding $n_{i}^{(t)}$, we consider that the bot interacts once every $\top_{i}^{(t)}$.

- The opinion can be calculated in a similar way using $\Theta_{i}^{(t)}$.

Once stated how the bot simulates a real user, we consider three experiment conditions, depending on whether of the value of the $P R^{(t)}$ is fixed or variable along the time, assuming the bot has answered the Hexad questionnaire previously (fixing $P R^{(0)}$ ). Moreover, we define how the error is computed in each case.

1. Case $\mathbf{A}$ - Constant $P R^{(0)}:$ A constant player rating, $P R^{(t+1)}=P R^{(0)}$, is assigned at any time. In this case, we use Equation 8 to calculate $U^{(t+1)}$ once and always use its maximal component.

We calculate the error as follows. Since $P R^{(t+1)}=P R^{(0)}$, the distance is simply calculated as $E r r=\left|R P T_{0}-P R^{(0)}\right|$. Note that if the user has answered the questionnaire accurately, we have $E r r=0$.

2. Case B - Random Dynamic $P R^{(t)}$ : A dynamic player rating $P R^{(t+1)}$ is randomly chosen at each time $t$ and then, the game element selected to be shown is also the maximal component of $U_{i}^{(t+1)}$. Note that this case iW equivalent to pick a random game element.

In this case, we compute the error as the average distance between $R P T_{0}$ and a random point $p \in\left\{\left(x_{1}, \ldots, x_{7}\right) \in[0,1]^{7}: \sum_{i=1}^{7} x_{i}=1\right\}$ using the Average Distance of Random Points in a Unit Hypercube ${ }^{4}$.

3. Case C - our method, Dynamic $P R^{(t)}$ : A dynamic player rating $P R^{(t+1)}$ is computed according the $s_{i}^{(t)}$ defined by Equation 6. Then, the bot simulates $\tau_{i}^{(t)}, n_{i}^{(t)}$, and $o_{i}^{(t)}$ using $\top_{i}^{(t)}$ and $\Theta_{i}^{(t)}$. The game element selected to be shown is a weighted random choice of $U^{(t+1)}$ (see step 3 in Figure 2).

We calculate the error based on the distances between $P R^{(t)}$ and $R P T_{0}$ for all $t$ from 1 to $n \_$iter, $E r r=\frac{1}{n \_ \text {iter }} \sum_{t=1}^{n \_ \text {iter }}\left|R P T_{0}-P R^{(t)}\right|$.

Experiments were performed using a homemade gamification sofware and run on Windows 10, Intel Core i7 processor with 8GB RAM. Table 1 shows the mean errors

\footnotetext{
${ }^{4}$ Average Distance of Random Points in a Unit Hypercube. https://martin-thoma.com/ curse-of-dimensionality/
} 
obtained in all the cases. In case $A$, as we take into account $P R^{(0)}=R P T_{0}$, accurate answers is the ideal case $(E r r=0)$. However, with inaccurate ones the error grows to 0.0311 . Moreover, both cases $B$ have similar errors $(0.08024$ and 0.08027$)$, because is a random selection of player type ratings and thus, they are almost independent of the reliability (accurate or inaccurate) of the answers. Finally, except in case $A$ with accurate

\begin{tabular}{l|c|c|c}
\hline Case & $\begin{array}{c}\text { A } \\
\text { Constant } P R^{(0)}\end{array}$ & $\begin{array}{c}\text { B - Random } \\
\text { Dynamic } P R^{(t)}\end{array}$ & $\begin{array}{c}\text { C - Our method } \\
\text { Dynamic } P R^{(t)}\end{array}$ \\
\hline \hline Accurate answers: $R P T_{0} \simeq P R^{(0)}$ & $0.08024(0.00052)$ & $0.0070(0.00166)$ \\
\hline Mean (SD) & 0 & 0.0804 & 0.0105 \\
Worse scenario of C & 0 & 0.0797 & 0.0029 \\
Best scenario of C & 0 & $0.08027(0.00040)$ & $0.0243(0.00475)$ \\
\hline \hline \multicolumn{5}{l}{ Inaccurate answers: $R P T_{0} \not P R^{(0)}$} & 0.0333 \\
\hline Mean (SD) & $0.0311(0.00404)$ & 0.012 & 0.0146 \\
Worse scenario of C & 0.0367 & 0.08018 & \\
Best scenario of C & 0.0233 &
\end{tabular}

Table 1. Table of mean errors, Err, when the questionnaire is answered accurately or inaccurately), together with the best and worst errors obtained in case $\mathrm{C}$.

answers, case $C$ behaves better than $A$ and $B$ even in its worse scenario $(E r r=0.0333)$.

In summary, we conclude that the case $A$ - where player type ratings are fixed along the experience - is the best when the users answer the questionnaire thoroughly and accurately, while our method, case $C$, works well in both cases (respond it either accurately or inaccurately). In fact, since it won't be possible to know whether (real) users answer the player type questionnaire accurately or not in real situations, our method is the most suitable for an adaptive gamified experience.

\section{Conclusions}

This research proposes a method to present the users game elements that fit their profile (player type). However, instead of taking a (static) picture of the profile at the beginning of the experience, we consider how it may change along the course of gamified activities. The method calculates the utility of showing a game element to the user based on the evolved Player Type (PT), the interactions on game elements, and the scores given by the users to those game elements. A bot simulated three different cases: constant player type ratings, random dynamic player type ratings, and dynamic player type ratings, where player type is recomputed using player's interactions, opinions at each step of the iterative method. The results show that our method achieves a low error considering both situations: when the user answers the player type questionnaire accurately $(E r r=0.0070)$ and inaccurately $(E r r=0.0243)$. As future work we will test the method with users and incorporate new inputs to our method such as emotions and activities completion.

\section{References}

[1] M Andrzej. Even Ninja Monkeys Like to Play: Gamification, Game Thinking and Motivational Design, 2015. 
[2] Richard A Bartle. Hearts, Clubs, Diamonds, Spades: Players Who Suit Muds Want more papers like this? Journal of MUD research, 1(1):19-undefined, 1996.

[3] S. Borges, R. Mizoguchi, V. H S Durelli, I. Bittencourt, and S. Isotani. A link between worlds: Towards a conceptual framework for bridging player and learner roles in gamified collaborative learning contexts. In Advances in Social Computing and Digital Education, pages 19-34. Springer, 2016.

[4] G. C. Challco, D. A. Moreira, R. Mizoguchi, and S. Isotani. An ontology engineering approach to gamify collaborative learning scenarios. In CYTED-RITOS International Workshop on Groupware, pages 185198. Springer, 2014.

[5] D Charles and M Black. Dynamic Player Modelling: A Framework for Player-centred Digital Games. Proceedings of 5th International Conference on Computer Games: Artificial Intelligence, Design and Education (CGAIDE'04), Microsoft(April):29-35, 2004.

[6] E. Chtouka, W. Guezguez, and N. B. Amor. Reinforcement learning for new adaptive gamified LMS. In International Conference on Digital Economy, pages 305-314. Springer, 2019.

[7] M. Denden, A. Tlili, F. Essalmi, and M. Jemni. Does personality affect students' perceived preferences for game elements in gamified learning environments? In Proceedings - IEEE 18th International Conference on Advanced Learning Technologies, ICALT 2018, pages 111-115, 2018.

[8] L. S. Ferro, S. P. Walz, and S. Greuter. Towards personalised, gamified systems. pages 1-6, 2013.

[9] O. Gené, M. Núñez, and A. Blanco. Gamification in MOOC: Challenges, opportunities and proposals for advancing MOOC model. ACM International Conference Proceeding Series, pages 215-220, 2014.

[10] S. Guggiari. Emergent Personalized Content in Video Games. (April), 2019.

[11] S. Hallifax, E. Lavoué, and A. Serna. To tailor or not to tailor gamification? An analysis of the impact of tailored game elements on learners behaviours and motivation. Lecture Notes in Computer Science, 12163 LNAI:216-227, 2020.

[12] S. Hallifax, A. Serna, J. Marty, and E. Lavoué. Adaptive Gamification in Education: A Literature Review of Current Trends and Developments. Lecture Notes in Computer Science, 11722 LNCS:294-307, 2019.

[13] S. Hallifax, A. Serna, J. Marty, G. Lavoué, and E. Lavoué. Factors to consider for tailored gamification. CHI PLAY 2019 - Proceedings of the Annual Symposium on Computer-Human Interaction in Play, pages 559-572, 2019.

[14] A.C.T Klock, I. Gasparini, M.S. Pimenta, and J. Hamari. Tailored gamification: A review of literature, volume 144. 2020.

[15] A. Knutas, J. Ikonen, D. Maggiorini, L. Ripamonti, and J. Porras. Creating student interaction profiles for adaptive collaboration gamification design. International Journal of Human Capital and Information Technology Professionals (IJHCITP), 7(3):47-62, 2016.

[16] . Lavoué, B. Monterrat, M. Desmarais, and S. George. Adaptive Gamification for Learning Environments. IEEE Transactions on Learning Technologies, 12(1):16-28, 2019.

[17] C. Lopez and C. Tucker. Towards personalized adaptive gamification. 2018.

[18] B. Monterrat, E. Lavoué, and S. George. Toward an Adaptive Gamification System for Learning Environments. In Computer Supported Education, pages 115-129, Cham, 2015. Springer International Publishing.

[19] A. Mora, G. F. Tondello, L. E. Nacke, and J. Arnedo-Moreno. Effect of personalized gameful design on student engagement. IEEE Global Engineering Education Conference, EDUCON, 2018-April:19251933, 2018.

[20] L. E. Nacke, C. Bateman, and R. L. Mandryk. BrainHex: A neurobiological gamer typology survey. Entertainment Computing, 5(1):55-62, 2014.

[21] A. Rapp, M. Alessandro, R. Simeoni, L. Console, and others. Playing while Testing: How to Gamify a User Field Evaluation. In Designing Gamification: Creating Gameful and Playful Experiences held in conjunction with SIGCHI Conference on Human Factors in Computing Systems (CHI'13). Gamification Research Network, 2013.

[22] J. Sabourin and J. Lester. Affect and Engagement in Game-BasedLearning Environments. IEEE Transactions on Affective Computing, 5(1):45-56, 2014.

[23] G. F. Tondello, R. Wehbe, L. Diamond, M. Busch, A. Marczewski, and L. E. Nacke. The Gamification User Types Hexad Scale Gustavo. CHI PLAY 2016 - Proceedings of the 2016 Annual Symposium on Computer-Human Interaction in Play, pages 229-243, 2016.

[24] DM-H Wen, D-J-W Chang, Y-T Lin, C-W Liang, and S-Y Yang. Gamification design for increasing customer purchase intention in a mobile marketing campaign app. In International conference on HCI in business, pages 440-448. Springer, 2014. 